Bio - grafía. Escritos sobre la Biología y su Enseñanza. ISSN 2027-1034

Edición Extraordinaria. p.p. 20 - 28

Memorias del IX Encuentro Nacional de Experiencias en Enseñanza de la Biología y la Educación Ambiental. IV Congreso Nacional de Investigación en Enseñanza de la Biología.

\title{
"ECOTROS" UNA PRÁCTICA DE CAMPO PARA LA ENSEÑ ANZA-APRENDIZAJE SOBRE ECOSISTEMAS Y REDES TRÓFICAS: UNA EXPERIENCIA CON ESTUDIANTES DE OCTAVO GRADO DE UNA INSTITUCIÓN EDUCATIVA OFICIAL DE LA CIUDAD DE NEIVA
}

\author{
"ECOTROS" A FIELD WORK FOR TEACHING AND LEARNING ABOUT \\ ECOSYSTEMS AND FOOD WEBS: AN EXPERIENCE WITH EIGHTH GRADERS FROM \\ AN OFFICIAL SCHOOL OF NEIVA CITY
}

\author{
Claudia del Pilar Auli Peña \\ Brenda Dayhana Delgado \\ Juan Felipe Herrera Polania \\ William Gentil Trujillo Solano \\ Luzmar Nidia Vargas Acosta \\ Elías Francisco Amórtegui Cedeño
}

\section{RESUMEN}

Presentamos resultados preliminares de la sistematización del diseño, desarrollo y evaluación de una Práctica de Campo para la enseñanza-aprendizaje de los conceptos de Ecosistema y Red Trófica, con estudiantes grado octavo de una Institución Educativa Oficial de la ciudad de Neiva (Huila-Colombia); esto en el marco del espacio académico de Didáctica I llevado a cabo en el periodo 2016-B del Programa de Licenciatura en Ciencias Naturales: Física, Química y Biología de la Universidad Surcolombiana (NeivaHuila). Realizamos la experiencia en tres momentos diferentes, una etapa de indagación de ideas previas del alumnado, una segunda con la presentación de la temática a través de actividades como discusiones de fragmentos de películas, la presentación de la guía de campo dirigida a la huerta escolar y al parque bosque próximo a la escuela y una tercera actividad a manera de socialización y evaluación de los saberes construidos de manera individual y grupal. Concluimos la importancia de las Prácticas de Campo como estrategia de aprendizaje de la Biología que permite el contacto directo con el fenómeno viviente y su potencialidad en la construcción de conocimientos, habilidades y actitudes sobre la conservación de la biodiversidad, así como en el entendimiento de la dinámica de los ecosistemas, específicamente sobre las redes tróficas, tomando como objeto de estudio, el propio espacio natural inmediato de la institución educativa.

PALABRAS CLAVES: ecosistema, redes tróficas, salidas de campo.

\footnotetext{
${ }^{1}$ Estudiantes del Programa de Licenciatura en Ciencias Naturales: Física, Química y Biología. Universidad Surcolombiana. Felipepolania19@gmail.com

2 Docente de Planta Tiempo Completo Universidad Surcolombiana, Facultad de Educación, Licenciatura en Ciencias Naturales: Física, Química y Biología, Neiva, Huila, Colombia. Elias.amortegui@usco.edu.co
} 
Bio - grafía. Escritos sobre la Biología y su Enseñanza. ISSN 2027-1034

Edición Extraordinaria. p.p. $20-28$

Memorias del IX Encuentro Nacional de Experiencias en Enseñanza de la Biología y la

Educación Ambiental. IV Congreso Nacional de Investigación en Enseñanza de la Biología.

\begin{abstract}
We present preliminary results of the systematization of the design, development and evaluation of a Field Practice for the teaching and learning of Ecosystem and Trophic Network concepts, with students from the eighth year of an Official Educational Institution of the city of Neiva (Huila); In the framework of the academic space of Didactics I carried out in the period 2016-B of the degree program in Natural Sciences: Physics, Chemistry and Biology of the Universidad Surcolombiana (Neiva-Huila). We conducted the experience in three different moments, a stage of inquiry of previous ideas of the students, a second with the presentation of the theme through activities such as discussions of movie trailers, presentation of the field guide (directed to the school garden And the forest park and a third activity in the form of socialization and evaluation of the individual and grouped knowledge. We conclude the importance of Field Practices as a learning strategy of Biology that allows direct contact with the living phenomenon and its Potential in the construction of knowledge, skills and attitudes on the conservation of biodiversity and understanding of the dynamics of ecosystems, specifically on trophic networks, taking as object of study the immediate natural space of the educational institution.
\end{abstract}

KEY WORDS: ecosystem, trophic networks, field trips.

\title{
INTRODUCCION
}

La Universidad Surcolombiana ofrece en el programa de pregrado, Licenciatura en Ciencias Naturales: Química, Física y Biología, el curso de Didáctica I en el cual se contempla la enseñanza y el aprendizaje de las ciencias naturales dentro de la dinámica del sistema de conocimientos y saberes. El curso se fundamenta en que la Didáctica de las Ciencias Naturales como contenido formativo de los maestros no se construye exclusivamente desde la teoría. En consecuencia, se busca establecer fuertes relaciones entre los referentes teóricos y la práctica; para ello, buena parte del abordaje del Seminario está centrado en el diseño y aplicación de una salida de campo.

Por otra parte las salidas de campo son una estrategia didáctica que es muy poco usada por las instituciones educativas, pues requieren un alto grado de preparación, organización y espacios adecuados para llevarlas a cabo. Las salidas de campo son un complemento adecuado para el desarrollo de la enseñanza-aprendizaje, pues ayudan a la fijación de los conocimientos a través de la vivencia y la experiencia directa. En el área de la biología las prácticas de campo sirven para crear conciencia ambiental, porque se desarrolla en un entorno específico, analizándose detalladamente sus componentes y aportes al ser humano, además la conciencia lograda en los estudiantes se aportara al medio sociocultural mejorándose el entorno (Amórtegui, Mayoral y Gavidia, 2017).

El trabajo práctico, incluye tanto salidas de campo como las experiencias en laboratorio, por tanto son usadas para descubrir y redescubrir los espacios en los cuales se 


\title{
Bio - grafía. Escritos sobre la Biología y su Enseñanza. ISSN 2027-1034
}

Edición Extraordinaria. p.p. 20 - 28

\author{
Memorias del IX Encuentro Nacional de Experiencias en Enseñanza de la Biología y la \\ Educación Ambiental. IV Congreso Nacional de Investigación en Enseñanza de la \\ Biología.
}

desenvuelven los estudiantes día a día, usando el método científico. Además permite conocer las percepciones de los estudiantes, facilitando el cambio conceptual, pues el estudiante debe argumentar y justificar los conocimientos que posee, por tanto también se puede usar como método de evaluación.

Las principales características del trabajo práctico es que se debe realizar por el estudiante, siempre se deben involucrar los procedimientos del método científico, con diferentes grados de aproximación según el nivel de los estudiantes. Para el desarrollo de las salidas de campo o trabajo práctico se requiere de material específico, que debe ser manejable por los estudiantes, por tanto debe ser sencillo. Y por último el trabajo de campo o práctico puede realizarse en cualquier lugar (Del Carmen, 2000) y (Barbera \& Valdes, 1996 ).

Para Amórtegui, Gavídia y Mayoral (2016), los docentes en ejercicio comparten de manera generalizada que la actividad de campo es fundamental para que los alumnos aprendan ciencias, aunque en sus prácticas esto esté ausente o se realice desde perspectivas didácticas tradicionales. Además de esto, actualmente existen varias normativas impuestas para la realización de salidas de campo, como los permisos, para conseguir salir con los estudiantes de la institución, seguros de vida, transportes y el aporte económico que deben realizar los padres de familia, que hacen más tedioso la organización y desmotivan al docente a la realización de salidas de campo. Es por ello que nosotros como docentes en formación de la Licenciatura en Ciencias Naturales de la Universidad Surcolombiana, cursando nuestro seminario de Didáctica I, nos dirigimos a una institución educativa oficial de Neiva, para realizar una salida de campo, en la que se pudiera evidenciar la construcción de conocimientos, habilidades y actitudes por medio de la observación, la indagación y la investigación del alumnado sobre el entorno de la misma institución.

\section{METODOLOGIA}

El trabajo investigativo se llevó a cabo en el segundo semestre del 2016, con un enfoque de investigación cualitativo, que se adelantó en tres etapas en los que se proponían objetivos específicos para el óptimo desarrollo y finalización de las fases. El primer paso del trabajo consistió en la selección de la temática a desarrollar relacionada con ecosistema y redes tróficas en estudiantes de octavo grado, elaborando un documento en el cual se tuvo en cuenta el contenido de los temas llevadas a cabo, el contexto de la problemática en relación hacia al tipo de población al cual iba dirigido, los objetivos actitudinales, conceptuales y procedimentales, las finalidades de aprendizaje, el aporte a la formación de futuros docentes en ciencias naturales y la justificación acerca de la temática.

La segunda fase se lleva acabo teniendo en cuentas las correcciones y observaciones de la primera parte del trabajo, y adicionalmente, se realiza la elaboración de un cuestionario sociodemográfico y un cuestionario de indagación de ideas previas de la temática, para la caracterización de la población de estudio. Además la realización de las respectivas guías 
Bio - grafía. Escritos sobre la Biología y su Enseñanza. ISSN 2027-1034

Edición Extraordinaria. p.p. 20 - 28

Memorias del IX Encuentro Nacional de Experiencias en Enseñanza de la Biología y la Educación Ambiental. IV Congreso Nacional de Investigación en Enseñanza de la Biología.

de campo a desarrollar con los estudiantes en las salidas y preparación de dicha actividad, teniendo en cuenta los estándares básicos de competencias del Ministerio de Educación Nacional (MEN), los materiales necesarios a implementar, el modelo de enseñanza, la relación teórica-práctica con respecto al tema y la evaluación del aprendizaje.

Por último en la fase final se lleva a cabo la presentación de los resultados y su respectivo análisis de la actividad realizada en la institución educativa, dando a conocer mediante fotografías, ilustraciones, escritos y videos por parte de los estudiantes, Por otra parte se hace la elaboración de un escrito reflexivo de la actividad realizada con los estudiantes, resaltando el aporte de este tipo de trabajos a la formación docente. De igual manera surge la necesidad de destacar los aspectos a mejorar y posibles modificaciones al trabajo realizado. La síntesis de la metodología se resume en la Tabla N. 1 en donde se puede detallar las actividades.

Cabe resaltar que cada una de las fases descritas anteriormente cuenta con un registro de video.

La población de estudio consistió en un grupo de 32 estudiantes de octavo grado de edades que oscilan entre 13 y 15 años, de estrato 1,2 y 3, de la Escuela Normal Superior de Neiva.

\section{RESULTADOS Y DISCUSIÓN}

\section{Primera Fase: Ideas previas de los estudiantes e introducción a la salida de campo}

El cuestionario de ideas previas aplicado a los estudiantes, no solo sirvió como instrumento de indagación para analizar los conocimientos de estos, sino que también permitió analizar la coherencia y relación de conceptos ecológicos y situaciones de interés local, regional y global. Para ello se hizo énfasis en las respuestas de los estudiantes $(R D E)$ en tres preguntas:

- Podrías explicar ¿Qué es una cadena trófica?, sustenta tu respuesta con un dibujo

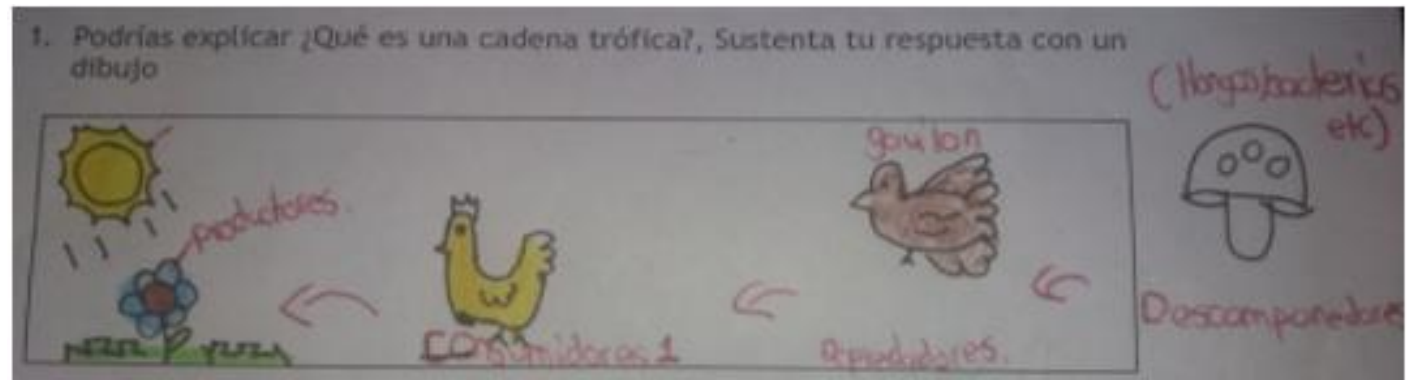

Figura No.1. llustración de la respuesta de un estudiante. RDE1 
Bio - grafía. Escritos sobre la Biología y su Enseñanza. ISSN 2027-1034

Edición Extraordinaria. p.p. 20 - 28

Memorias del IX Encuentro Nacional de Experiencias en Enseñanza de la Biología y la Educación Ambiental. IV Congreso Nacional de Investigación en Enseñanza de la Biología.

De los estudiantes encuestados el resto, presentó inconvenientes al plasmar gráficamente la respuesta, por lo que dejaron en blanco el espacio.

- ¿Qué factores intervienen en un sistema trófico?:

$>$ "Los que intervienen somos nosotros los humanos. Porque nosotros mismos nos encargamos de cazar ciertos animales, haciendo que se vayan extinguiendo". RED3.

> "Intervenimos nosotros los humanos, porque nosotros nos encargamos de cuidar cualquier clase de animal, porque ellos también necesitan de nosotros". RDE20.

> "Creo que lo que interviene es la bota de basura porque esto puede impedir la cadena tróficas". RDE 13.

$>$ "intervienen factores vivos como los no vivos el aire, el calor y otros". RDE25.

Se aprecia que priorizan al hombre como factor principal en la intervención de un sistema trófico; pero lo vinculan de manera negativa en la mayoría de los casos ya que lo relacionan con destrucción y contaminación. Por otra parte, una minoría de estudiantes los relaciona de manera mucho más generalizada vinculándolos como factores bióticos y abióticos que intervienen en dichas cadenas.

- ¿Crees que la represa el Quimbo afecto las cadenas tróficas?, ¿Cómo?, ¿Por qué?; su respuesta siempre fue afirmativa, sin embargo, se justificaban de forma diferente:

> "si, porque antes de existir el "quimbo" existían más animales en ese espacio "flora y fauna". Como afecto: destruyendo el habitad de esos animales y porque: porque este embalse será llenado de agua. RDE15.

"si esto afecta a las cadenas tróficas porque los quimbos al inundar todas estas hectáreas morirán muchos animales de que son las presas de otros animales". RDE10.

Esta primer actividad se realizó como punto de partida, pues se considera importante sobrepasar los postulados tradicionales en los que se concibe a los alumnos como agentes pasivos y tabulas rasas; a diferencia de esto, aquí cobran gran importancia los alumnos en la organización, evaluación de los contenidos y las actividades de enseñanza (Valbuena, 2007) y por tanto requieren actividades para su detección, activación, confrontación y reestructuración, en este caso abordar sus ideas sobre los ecosistemas y redes tróficas.

En concreto, en esta actividad se empezó a despertar el interés de los alumnos en la apropiación de los espacios que los rodean y la concientización de la problemática ambiental y así tomar decisión sobre la defensa de los ecosistemas (Álvarez, 2004). 
Bio - grafía. Escritos sobre la Biología y su Enseñanza. ISSN 2027-1034

Edición Extraordinaria. p.p. 20 - 28

Memorias del IX Encuentro Nacional de Experiencias en Enseñanza de la Biología y la Educación Ambiental. IV Congreso Nacional de Investigación en Enseñanza de la Biología.

Para centrar los saberes de los educandos, se observaron fragmentos de películas como "Avatar" en las que, junto con el docente, se ponían en discusión problemáticas sobre el cuidado de los ecosistemas; en este punto se evidenció una colaboración y buena disposición de los estudiantes durante el conversatorio, cabe resaltar que en este punto se pudo detallar una postura más coherente en la expresión de sus ideas.

Realizar esta actividad desde la perspectiva de Grilli (2016) quien afirma que la televisión, las series de ciencia ficción y el cine pueden ser empleados como estrategia de enseñanza de las ciencias naturales, pues permiten comprender el mundo desde la vida cotidiana, motiva al alumnado y admite la vinculación escuela-sociedad.

Para finalizar esta primera fase tuvo como finalidad aclarar algunos conceptos ecológicos en los estudiantes y prepararlos para el desarrollo de la salida de campo.

\section{Segunda Fase: Desarrollo de las salidas de campo}

Durante el desarrollo de la salida de campo el educando contó con una guía, en la cual se indicaban los elementos que se considera toda guía de campo debería comprender:

Temática ¿Cuál será la temática biológica a tratar en la salida?

Lugar ¿A dónde iremos?

Curso: ¿Quiénes iremos?

Duración: ¿Cuánto tiempo trabajaremos allí?

Motivación ¿Cuál es la importancia del lugar a visitar?

Indagación de ideas previas ¿Qué sabes?

Planteamiento de Contenidos ¿Qué aprenderás?

Trabajo de campo ¿Qué harás en el campo? ¿Cómo lo harás?

Finalización ¿Cómo concluimos la actividad? ¿Cómo comunico mis aprendizajes?

En la Figura No.2. Se muestra algunas de las anotaciones en las guías de campo por parte de los estudiantes durante la salida.




Bio - grafía. Escritos sobre la Biología y su Enseñanza. ISSN 2027-1034

Edición Extraordinaria. p.p. 20 - 28

Memorias del IX Encuentro Nacional de Experiencias en Enseñanza de la Biología y la Educación Ambiental. IV Congreso Nacional de Investigación en Enseñanza de la Biología.

Tal como lo evidencia la Figura No.3 se aprecia un interés del educando por querer conocer y saber más del entorno que le rodea, de acuerdo con muchos didactas, el estímulo ha sido siempre imprescindible para que haya una consolidación en el aprendizaje y tal como se pudo apreciar durante el desarrollo de la salida de campo, este fue un factor fundamental, en el que los estudiantes se apropiaron y pudieron vivenciar de una forma más amena lo contenidos teóricos.
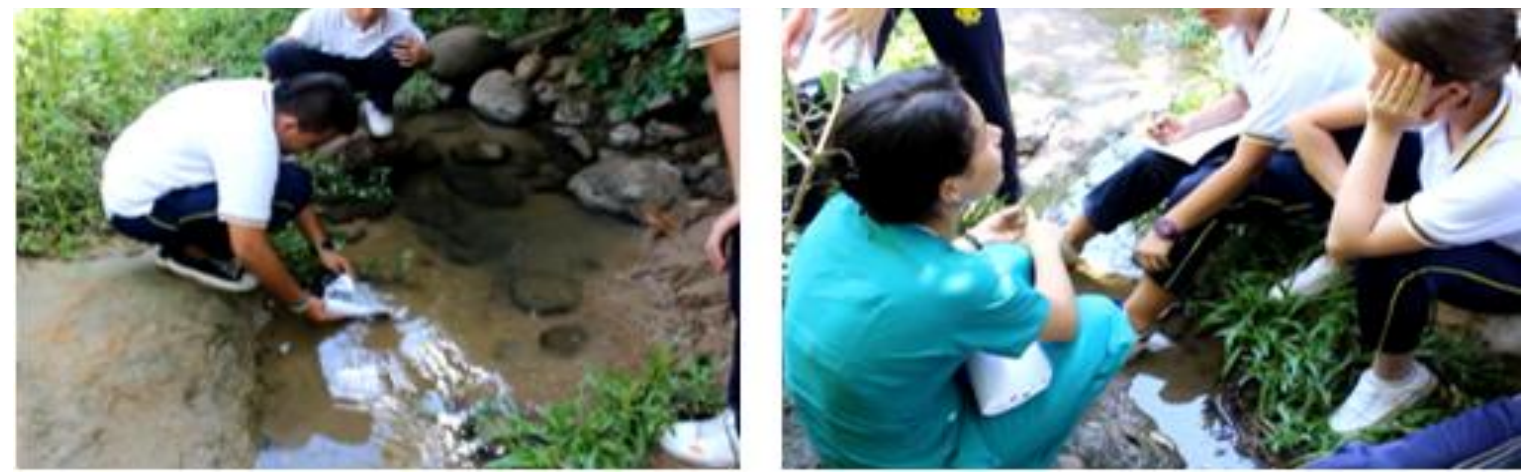

Figura No. 3. Salida de campo al Riachuelo la Cabuya.

Dadas estas circunstancias cabe aclarar de cierta forma la precisión que tienen desde su opinión Rodriguez \& Amortegui, (2013), donde expresan "...la salida de campo como un recurso pedagógico... suministra al estudiante una interacción con la naturaleza en forma directa, fundamenta los conocimientos y posibilita la apropiación de los temas".

\section{Tercera Fase: Evaluación de las salidas de campo como modelo de enseñanza- aprendizaje.}

Pese a que la evaluación en el educando fue constante, la adquisición y apropiación de los conceptos fue consolidada durante la tercera fase, en la que se impartió un diálogo crítico donde los estudiantes situaron y realizaron de manera adecuada una red trófica característica de un tipo de ecosistema, sin pasar por alto la presencia de los factores bióticos y abióticos.

Tal como lo ilustra la Figura No.4 y como lo dijo explícitamente un estudiante, "La salida de campo me logró dar a conocer los diferentes ecosistemas y entender el equilibrio que se presenta en un entorno y habitad determinado". RDE1. De igual forma como lo expresó otro estudiante, "esta salida me permitió contrastar conceptos previos a la actividad, en relación a los factores abióticos presentes en los ecosistemas visitados durante las salidas". RDE2 
Bio - grafía. Escritos sobre la Biología y su Enseñanza. ISSN 2027-1034

Edición Extraordinaria. p.p. 20 - 28

Memorias del IX Encuentro Nacional de Experiencias en Enseñanza de la Biología y la Educación Ambiental. IV Congreso Nacional de Investigación en Enseñanza de la Biología.

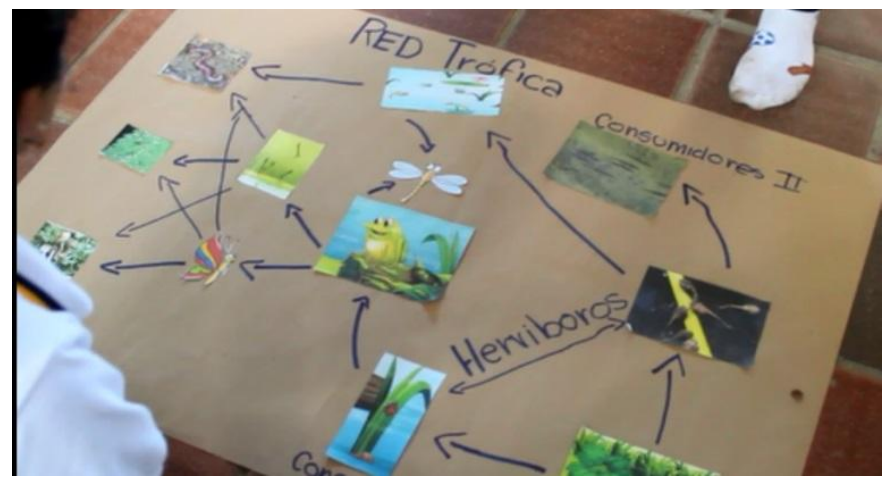

Figura No.4 Cadena trófica realizada por un de los grupos del curso.

\section{CONCLUSIONES}

Al emplear las salidas de campo como estrategia de enseñanza, el estudiante pone de manifiesto sus ideas previas, interés, gustos y capacidades, que ha aprendido de manera cotidiana a través de su vida y que les permite de manera popular explicar la lógica de los sucesos que observan; por esta razón las salidas de campo complementan, ajustan, consolidan los conceptos con lo que viene el educando. No obstante, al emplear este modelo de enseñanza se logra evidenciar una mejora considerable en la aclaración, construcción y apropiación del conocimiento, sin embargo, el comportamiento del estudiante se ve influenciado, cuando estos quieren explorar por cuenta propia sin necesidad del acompañamiento del docente.

Es de vital importancia generar este tipo de actividades en las cuales se puedan desarrollar diferentes temáticas en relación con la enseñanza de las ciencias, ya que facilita la apropiación del conocimiento científico y mediante una participación activa y en el cual se le brinde un ambiente óptimo al estudiante, logre dar a conocer las habilidades y destrezas argumentativas, constructivas, críticas, entre otras; que logren fortalecer e incentivar un sentido de pertenencia por la flora y fauna del municipio, del departamento y del país.

Cabe además resaltar que pese a la gran dificultad de realizar salidas de campo debido a la diversidad de elementos que demandan de su preparación, desarrollo y conclusión es de fundamental importancia que los maestros los lleven a cabo en su práctica docente. Es por esta razón que el desarrollo de la salida de campo como unidad temática, no solo permitió como futuros docentes consolidar y argumentar de una forma distinta los componentes teóricos, sino que generó al estudiante una seguridad para preguntar sucesos que no puede captar fácilmente en una clase magistral, de esta forma como docentes en formación se pudo analizar que existe una represión biunívoca entre el 
Bio - grafía. Escritos sobre la Biología y su Enseñanza. ISSN 2027-1034

Edición Extraordinaria. p.p. 20 - 28

Memorias del IX Encuentro Nacional de Experiencias en Enseñanza de la Biología y la Educación Ambiental. IV Congreso Nacional de Investigación en Enseñanza de la Biología.

aprendizaje y la motivación por querer conocer más, cuando el educando se encuentra confinado en un aula de clase.

Por ultimo observando las expresiones de los estudiantes es imprescindible no olvidar que hay que asombrar a los estudiantes para estimular el querer aprender.

\section{REFERENCIAS BIBLIOGRÁFICAS}

Álvarez, R. P. (2004). Formación Superior Basada En Competencias De Interdisciplinariedad Y Trabajo Autónomo Del Estudiante. Iberoamericana de Educación.

Amórtegui E. F., Gavídia V. y Mayoral O. (2017). Aportaciones de las Prácticas de Campo en la formación del profesorado de Biología: un problema de investigación y una revisión documental. Didáctica de las ciencias experimentales y sociales. 37

Barbera, O., y Valdés, P. (1996). El Trabajo Práctico en la Enseñanza de las Ciencias.

Boya Busquet, M. (2008). ¿Integrar conservación y desarrollo? Usos y representaciones sociales de la naturaleza en el parque natural Obô de Sao Tomé. Patrimonialización de La Naturaleza. El Marco Social de Las Políticas Ambientales. Retrieved from http://www.ankulegi.org/wp-content/uploads/2012/03/0906Boya-Busquet.pdf

Del Carmen, L. (2000). Los trabajos prácticos. En Perales, J. y Cañal, P. (coord) Didáctica de las ciencias experimentales. Editorial Marfil Alcoy: España.

Grilli, J (2016). Cine de ciencia ficción y enseñanza de las ciencias. Dos escuelas paralelas que deben encontrarse en las aulas. Revista Eureka sobre Enseñanza y Divulgación de las Ciencias. 13 (1), 137-148.

Rodríguez, C., y Amórtegui, E. (2013). Prácticas de campo como estrategia de enseñanza de la biodiversidad en alumnos de básica secundaria del colegio Champagnat: planteamiento del problema. Revista Virtual EDUCyT.

Valbuena, E. (2007). El Conocimiento Didáctico del Contenido Biológico. Estudio de las concepciones disciplinares y didácticas de futuros docentes de la Universidad Pedagógica Nacional (Colombia) (Tesis de Doctorado). Universidad Complutense de Madrid, Madrid, España. 